\title{
Contextualism and the Principle of Tolerance
}

\begin{abstract}
When we bring together certain plausible and compatible principles guiding the use of vague predicates the inclination to accept that vague predicates are tolerant is significantly weakened. As the principle of tolerance is a troublesome, paradox inducing principle, a theory giving a satisfactory account of the nature of vague predicates and accounting for the appeal of the sorites paradox, without recourse to the principle of tolerance is a worthy addition to the vagueness debate. The theory offered, Contextual Intolerance, draws considerably on Sainsbury's (1996) thesis of the boundarylessness of vague concepts and on the contextualist theories of vagueness offered by Stewart Shapiro (2003, 2006) and Diana Raffman (1994).
\end{abstract}

\section{Introduction}

The motivating intuition behind the principle of tolerance is that vague predicates are such that, if the predicate applies to an object $n$, then the predicate must also apply to an object that is suitably similar to $n$ in the relevant respect. A definition of what it takes for a predicate, 'is $F$ ' to be tolerant was first given by Crispin Wright (1976):

Standard Tolerance: Whereas large enough differences in $F$ 's parameter of application sometimes matter to the justice with which it is applied, some small enough difference never thus matters.

If we stipulate that a vague predicate, e.g. 'is bald', is such that standard tolerance governs competence with use or is at the very least a semantic default, then under certain sorites-prone conditions standard tolerance shows itself to be a troublesome, paradox inducing, principle. An example of sorites-prone conditions for 'is bald' is a series of 100,000 men, where \#1 has no hairs on his head, \#100,000 has 99,999 hairs and each man in the series has exactly one more hair than his predecessor. 
The standard version of the sorites paradox sees a false conclusion reached from apparently true premises via apparently valid reasoning. Consider this example ranging over the series of 100,000 men, where $F$ schematises the vague predicate 'is bald"1,

Base step: $\quad F 1$

Universal Generalisation: $\quad(\forall x)\left(F x \rightarrow F x^{\prime}\right)$

Conclusion: $\quad F 100,000$

The base step repesents the fact that the first man in the series is bald. The universal generalisation represents our tolerance intuition that, for any object that satifies the predicate 'is bald', an object which is relevantly similar will also satisfy the predicate. Elemetary reasoning via universal instantiation and modus ponens leads to the conclusion $F 100,000$, yet a man with 99,999 hairs clearly does not satisfy the predicate 'is bald', hence the paradox.

Contextualist solutions to the sorites rely on some form of what Akerman and Greenough (2010) call weak tolerance;

Weak Tolerance: It is not the case that: there is a context of utterance $\mathrm{C}$ and there is an $\mathrm{x}$ such that $\mathrm{x}$ and $\mathrm{x}$ ' are considered together as a pair by a single subject in $C$

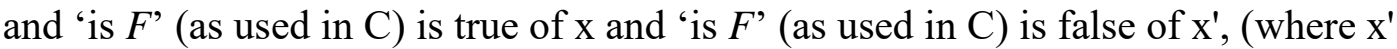
is adjacent to $\mathrm{x}$ in the sorites series running from $F$ to not- $F$ ).

Weak tolerance, henceforth simply tolerance, has been brought to bear by contextualists in different forms. Diana Raffman represents tolerance with IP*, 
IP*: for any $n$, if patch \#n is red then patch \#(n+1) is red, relative to a pairwise presentational context' $(1994,68)$

Delia Graff Fara represents tolerance with Salient Similarity (SS),

SS: if two things are saliently similar, then it cannot be that one is in the extension of the predicate, or in its anti-extension, while the other is not $(2000,57)$.

And Stewart Shapiro gives the weak contextualist version of Wright's tolerance principle (CT),

CT: suppose that two objects $a, a^{\prime}$ in the field of $P$ differ only marginally in the relevant respect (on which $P$ is tolerant). Then if one judges $a$ to have $P$, then she cannot competently judge $a^{\prime}$ in any other manner [within in the same conversational context]. (2006: 8$)$

The contextual theorists propose that through context dependent judgements and context shifts they can give a solution to the paradox that has the desirable features of preserving the principle of tolerance and providing an explanation of the seductive appeal of the paradox. ${ }^{2}$

Here it is proposed that the context shifts required to provide this package are otherwise unmotivated and ad hoc. Shapiro semantic solution to the paradox, arguably the most robust of the contextualist theories, is the focus of the specific criticisms but the general challenge - that non-standard (i.e. non-Kaplanian) contextual mechanisms must be motivated independently of the fact that they may provide a solution to the sorites paradox - applies to all extant contextualist theories of vagueness. ${ }^{3}$ Moving towards a positive contextualist account that requires no ad hoc context shifts, it is 
proposed that the status of the principle of tolerance as a principle governing the competent use of vague predicates is significantly weakened when it is positioned in a contextualist framework. Vagueness is to be characterised not by tolerance but by elements of permissibility operating within a context.

\section{Contextualism and Boundarylessness}

Contextualist theories rely heavily on what are known as 'forced-march' versions of the sorites. In the forced-march sorites, paradox arises when we imagine a judger progressing along a sorites-prone series, like the series of 100,000 men described above, and passing judgements. The judgements are constrained by two incompatible principles that appear to govern the competent use of vague predicates. The first principle states that vague predicates are tolerant - as above - and the second states that a competent judger must respect what Delia Graff Fara (2000) termed the clearcase constraint.

Clear-case Constraint: For each predicate there will be only a limited range of cases which it will be permissible to count as positive instances and there will be a class of things which it will be mandatory to class as positive instances. (Fara: 2000, 57)

Consider the series of 100,000 men, proceeding from a clearly bald man (\#1) to a clearly not bald man (\#100,000), with each man in the series differing only marginally from his predecessor in terms of the number and arrangement of hairs. Assuming tolerance is in play, it is easy to see how the forced-march paradox will arise. In order to be competent one must respect the clear-case constraint, judging \#1 to be bald and $\# 100,000$ to be not bald and yet we are, we shall assume, guided by the principle of 
tolerance which states that at each stage, if $x$ is judged to be bald then $x$, if judged at all, must be judged likewise. Given these restraints, the forced-march game is one that we cannot win: if we respect tolerance we violate the clear-case constraint, if we respect the clear-case constraint we violate tolerance.

According to Shapiro, we must "jump" at some point when going through the series, from $x$ is bald to $x$ is not bald judgements, in order to avoid the incompetent claim that \#100,000 is bald - but the question is, how can we do this without violating tolerance ${ }^{4}$

First, we can narrow down the area where such a jump would be permissible, notwithstanding tolerance for the present. The series contains determinate cases for which the facts about an object $x$ (such as the number and arrangement of hairs) determine whether the conditions of application of the predicate have been met. In such cases it is determinate that a sentence, $S$, which asserts that $x$ has the property $F$ is either true or false. However, somewhere in the series there is a borderline case: a case for which it is neither determinate that $S$ is true nor determinate that $S$ is false. ${ }^{5}$ For any such case, competent assertions of $S$ are governed by the principle of 'opentexture':

Open-texture: In borderline cases, a speaker is free to assert ' $F x$ ' or ' $\neg F x$ ', without offending against the meanings of the terms, or against any other rules of language use. $(2006,10)$

Shapiro also employs the following response-dependence principle which determines the extension of the vague predicate: 
Judgement-dependence: Vague predicates are judgement-dependent (in the borderline area) such that an item lies in a given category iff the relevant subjects would judge it to lie in that category. $(2006,41)$

Shapiro incorporates the open-texture thesis into a conversational framework that allows for an assertion regarding an open case to be, not only assertable, but true in a context, where truth in $a$ context is distinct from truth in every context (determinate truth). This notion of truth in a context opens up a possibility. If Shapiro can show that a jump brings about a change in conversational context such that an assertion of $F x$ and an assertion of $\neg F x^{\prime}$ occur in different contexts, the jump can occur without violation of tolerance. Requiring some mechanism to regulate this proposed feature of language, Shapiro turns to Lewis's theory of conversational scorekeeping (Lewis, 1979).

Lewis claims that in any conversation a 'scorecard' will record such things as shared assumptions, details of the environmental context, salient objects or persons, comparison classes and other information that is required to determine which conversational moves are permissible. This record is necessary for determining the assertability conditions of a given statement within the conversation and evolves continually with each assertion or change in the environment.

Shapiro claims that this is just the framework that his context-change theory requires. If he can persuade us that a jump from the assertion of the sentence That $(x)$ is bald to the assertion of That $\left(x^{\prime}\right)$ is not bald brings about a change in conversational context and in particular that the addition of That ( $\left.x^{\prime}\right)$ is not bald to the conversational record 
removes the earlier That $(x)$ is bald, weak tolerance can be protected and the paradox avoided. ${ }^{6}$ The analogy with Lewis's use of the mechanism is given:

The event described here is quite similar to the outcome in one of Lewis's scenarios $[\ldots]$ In that story, the participants in a conversation first agree to a 'low' standard when they accept 'France is hexagonal'. Later, when they demur from 'Italy is boot shaped', the standard is raised, and so 'France is hexagonal' is implicitly removed from the record. Similarly, when the present conversationalists explicitly declare that \#975 is not bald, they implicitly retract the statement that \#974 is bald. In short, the conversational score is the device that enforces tolerance. $(2003,52)$

According to Shapiro, not only is number 974 is bald removed but also a 'backward spread' (Raffman, 1994) occurs removing an indeterminate number of earlier assertions from the conversational record. ${ }^{7}$ In any case, the removal of (at least) the earlier assertion, along with Shapiro's assumption that tolerance does not hold across contexts, allows that $x^{\prime}$ can be judged to fall outwith the extension of the predicate and $x$ judged to fall within the extension, without violation of tolerance and without compromising competence. The change of context accompanying the jump permits the judger to pass legitimately from assertions of the sentence That is bald to assertions of the sentence That is not bald within the borderline area.

Shapiro's solution to the forced-march sorites depends heavily on its being plausible that the context shifts in the way that he describes. However, while Lewis is explicit about which parameters shift, what causes them to shift and, most importantly, how 
participants in the conversation are aware of such shifts, Shapiro is silent. Shapiro's theory is at risk of appearing ad hoc.

Shapiro must motivate the view that an assertion of the sentence That is bald, of an object in the borderline area, and an assertion of the sentence That is not bald of an adjacent object creates an unacceptable tension on the scorecard. At first glance, such a tension does not seem obvious: there is nothing contradictory about That $(x)$ is bald and That ( $x$ ) is not bald. But perhaps the tension that Shapiro requires can be created.

What follows is not exegesis of Shapiro's theory but an attempt to search for the kinematics of scorekeeping in the context of a forced-march scenario. There are (at least) two possible approaches, both supported by some textual evidence, which Shapiro may consider to be obvious candidates.

Perhaps Shapiro assumes a tension is created on the scorecard between a presupposition and an assertion. This is a plausible option as it would account for Shapiro's assumption that the existing Lewisian framework is already equipped for his theory, given that the Lewisian framework is already equipped to accommodate presuppositions.

It may be that, given tolerance, when the objects in the series are connected by a similarity chain a competent assertion of 'Number 939 in the series is not bald' requires the presupposition that number 938 in the series is not bald, such that the assertion of 'Number 939 in the series is not bald' implicitly adds Number 938 in the series is not bald to the conversational record. This would create a tension between 
the presupposed Number 938 in the series is not bald and the asserted Number 939 in the series is bald. Shapiro alludes to this $(2003,52)$ :

In declaring man 975 to be not bald, they implicitly deny that 974 is bald, and so 'Man 974 is bald' is removed from the conversational record.

To set up the first problem for this approach suppose that there are two incompatible (by Shapiro's lights) assertions (as opposed to an incompatible assertion and presupposition) on the scorecard; Number 938 in the series is bald and Number 939 in the series is not bald. According to Shapiro, the latter assertion causes a context shift that wipes the earlier assertion (plus a few more) off the scorecard, hereby preserving tolerance. But why should we suppose that it is the latter assertion that dominates the scorecard and not the earlier one? The fact that there are 'incompatible' assertions on the scorecard gives no notion of priority. ${ }^{8}$ Neither the scorecard nor the principle of tolerance tells us whether we are in a context in which we are using the predicate bald $_{c}$ or a context in which we are using the predicate bald $c^{\prime}$, all we know is that we cannot be employing both predicates in the same context. So, while Shapiro assumes that the earlier assertions are wiped off, the scorecard would be just as efficient in protecting tolerance were it to override the latter assertion. ${ }^{9}$

This problem of direction may seem insignificant but it arises with force with respect to a conflict between an assertion and a presupposition. The scorecard contains the asserted Number 938 is bald. When the jump occurs and 'Number 939 is not bald' is asserted we are to suppose that the presupposition Number 938 is not bald is registered on the scorecard. The result is that we have a clash between the asserted 
Number 938 is bald and the presupposed Number 938 is not bald. Nothing about the scorecard or the principle of tolerance dictates which of these must change. Furthermore, the items in 'conflict' are an assertion and a presupposition. Shapiro's view requires that the presupposition would trump the assertion on the scorecard, yet it seems that we have no principled reason for favouring a conflicting presupposition over an assertion. ${ }^{10}$ So, even if Shapiro could explain how and why later assertions can override earlier assertions, we would need a further argument from Shapiro to support the claim that later presuppositions can override earlier assertions.

The second problem with the presupposition response is that, unless Shapiro offers a distinct theory of presupposition, the relationship between the assertion and the proposed presupposition is not the correct one. According to Stalnaker (whose theory Lewis acknowledges as the basis for his own scorekeeping account of presupposition), a sentence $P$ presupposes $Q$, if $Q$ is required by both the assertion of $P$ and the assertion of $\neg P .{ }^{11}$ Consider the following example,

P: The Queen of England is rich.

Both the assertion that $P$ and the denial of $P$ require the presupposition, $Q$, for assertibility or truth.

Q: There is one and only one current Queen of England.

If we look back now at the sorites case we can see that this relationship does not hold between the asserted

R: Number 975 is not bald,

and the posited presupposition 
S: Number 974 is not bald.

That is, it is not the case that both the assertion that $R$ and the assertion that $\neg R$ require the presupposition $S$. What Shapiro needs, rather, is that the assertion $R$ requires the presupposition $S$ and the assertion that $\neg R$ requires the presupposition $\neg S$. Therefore 'Number 974 is not bald' does not have the correct relation to the asserted 'Number 975 is not bald' for it to be implicitly placed on the conversational record as a presupposition. The relation that Shapiro needs between $R$ and $S$ is that of mutual entailment, not presupposition. But entailment is not available to him.

In searching for the kinematics of scorekeeping in order to bolster the plausibility of Shapiro's scorekeeping mechanism, it seems that we can discount the option of creating a tension between an assertion and a presupposition.

Perhaps the scorekeeping account is to be supported by our dispositions to judge: the earlier assertion 'Number 974 is bald' should be removed from the conversational record when 'Number 975 is not bald' is asserted because at that point in the conversation the judger would be disposed to deny that number 974 is bald, were he asked. Recalling the phenomenon of backward spread, not only would the judger be disposed to deny that number 974 is bald but he would also be disposed to deny that some of the proceeding members of the series are bald, so the corresponding earlier assertions (a vague amount of them) would be removed from the conversational record. Shapiro seems to support this dispositional account $(2003,52):{ }^{12}$ 
We assume that man \#975 is the first 'jump' [...] Suppose that we explicitly ask them about \#974 again, after reminding them that they just called that man 'bald', and that they can barely distinguish \#974 from \#975 (if at all). [...] I'd speculate that they would explicitly retract that judgement, saying that \#974 is not bald [...] Just as 'Man 974 is bald' comes off the record, so does 'Man 973 is bald'; ditto for a few more of their recent pronouncements.

First off we can note that, by going this way, Shapiro avoids the problem of direction as, in this case, the jump itself brings about a change in dispositions regarding the earlier judgements.

However, there is something left unexplained here. Shapiro has stated that, for any competent judger, an assertion regarding an object in the series is accompanied by a disposition to co-classify the objects on either side. ${ }^{13}$ At the same time, Shapiro claims that to retain competence a judger must jump at some point throughout the series; in order to do so it is clear that the judger must momentarily override his disposition to co-classify (the tolerance disposition). Yet tolerance must be immediately reinstated via the judger's dispositions as soon as the jump has occurred. That is, is not simply that the tolerance disposition reasserts itself with regard to future judgements but also with regard to recently asserted judgements. What is it that makes the tolerance disposition reassert itself, adjusting recently asserted judgements, the very instant a jump is made ${ }^{14}$ The apparatus is left unexplained.

That worry aside, how does the claim that the conversational record reflects our dispositions fit with the semantic principles of tolerance and judgement-dependence? 
A dispositional version of tolerance would state that a (competent) judger who asserts 'Number 975 is not bald' is disposed to deny that number 974 and number 976 are bald. But note that the linguistic version of backward spread that Shapiro employs goes beyond dispositional-tolerance, as stated. What Shapiro requires is some form of extended dispositional-tolerance: a (competent) judger who asserts 'Number 975 is not bald' would be disposed to assert ' $x$ is not bald' of a few of the objects each side of number 975. This extended dispositional principle will show itself in a dispositional version of Shapiro's judgement-dependent principle. Shapiro's judgement-dependent principle states that an object satisfies a vague predicate iff it is (competently) judged to have $F$. But if Shapiro is committed to the dispositional version of this principle i.e., that an object satisfies a vague predicate iff a competent judger is disposed to judge it to satisfy the vague predicate, then a serious problem arises. ${ }^{15}$ The guiding principle behind Shapiro's theory is that tolerance can be preserved if a jump marks, not a boundary, but a change in conversational context. But now it seems that, when a jump occurs, the dispositions of the judger register on the scorecard, causing a boundary to appear. Consider a judger who, having asserted 'Number 974 is bald', jumps and asserts 'Number 975 is not bald'. According to Shapiro, at least one of the earlier assertions is removed from the conversational record, in accordance with a change in the judger's dispositions. Shapiro supposes that his weak tolerance principle will keep him out of trouble here; if (at least) 'Number 974 is bald' is removed, number 974 is unjudged and the principle of tolerance holds. But if in order to remove the asserted 'Number 974 is bald' the scorecard must register the judger's new disposition that number 974 is not bald, then 'Number 974 is not bald' registers on the scorecard and tolerance is violated: the scorecard now registers that number 973 is bald and that number 974 is not bald. The 
extended versions of the principles that accommodate the phenomenon of backwards spread do not help as they simply place the cut-off further down the series. ${ }^{16}$

Shapiro states that the 'conversational score is the device that enforces tolerance' but it is not clear what that amounts to. If Shapiro means that an attempted violation of the principle of tolerance causes the context to shift, we are still lacking any account of the kinematics of scorekeeping that supports this stipulation. In the standard Lewisian cases we explain apparent incompatibility away by saying that the value taken by some parameter can legitimately vary across the two contexts; we have one variable standard governing permissible assertions. In the Lewisian case a variation in standard marks a change in context; furthermore, no single context could be both a high and low standard context. In the sorites case, by contrast, it is not that some parameter that has shifted during the conversation but rather that there are two incompatible principles governing permissible assertions in each context; the clearcase constraint and the principle of tolerance. The stipulation of the context shift that is required to preserve tolerance in a context is entirely ad hoc. It is difficult to see how to make the conversational mechanism work, except by stipulation.

\section{Contextual Intolerance}

The contextualists stipulate context shifts to preserve tolerance while accommodating the clear-case constraint. But why think that the principle of tolerance is a restriction on competence with a context dependent vague predicate in any case?

If tolerance is in play during a forced-march, judgers have two options: they can either respect tolerance and judge a clearly not bald man to be bald or they can violate 
tolerance and, for some adjacent members, judge the first but not the second to lie in the extension of the predicate, while judging the clear cases correctly.

Regarding the first option, as Shapiro rightly states, 'That way lies madness (or at least incompetence)' (2003, 51). A judger who continues right through the series and judges a man with a full head of hair to be bald has displayed incompetence with the term 'bald'. And notice that offering up the principle of tolerance as justification for his judgement in no way eradicates or lessen this incompetence.

Regarding the second option, violating tolerance, what lies that way? Certainly not madness nor, it will now be pressed, even incompetence. ${ }^{17}$ Consider the forced march scenario again and imagine a judger who, upon jumping, is not inclined to retract his earlier judgements, instead insisting that \#974 is the last bald man and \#975 the first non-bald man within that context. Would we judge such a judger to be incompetent? The judger takes the inclusion of the polar cases to require him to judge the last bald man and first non-bald man to be somewhere in the borderline area. To reason in such a manner within any sorites context would be to reason competently so why are we assume that tolerance is the semantic default?

It will prove useful when considering vague predicates to think of them not in negative terms nor in terms of restrictions that may accompany competent usage, but in terms of permissibility; what are we permitted to do when applying vague terms? It is in this spirit that Mark Sainsbury (1991) offered the slogan "vagueness offers freedom". 
Sainsbury proposes that vague predicates correspond to vague concepts which are themselves concepts without boundaries. In contrast to a concept being tolerant, a concept being boundaryless does not preclude boundaries being drawn. While boundaryless concepts need not draw boundaries it does not follow that they must not draw boundaries. To think that the boundarylessness of vague concepts is restrictive in this way is to make a faulty inference.

A boundaryless concept is one which, for closely similar pairs, never makes it mandatory to apply the concept to one member of the pair, and withhold it from the other; hence, the argument runs, a boundaryless concept is one which, for closely similar pairs, makes it mandatory never to apply the concept to one member of the pair, and withhold it from the other. The inference depends upon the move from something being not mandatory to its being forbidden; a move legitimate within the totalitarianism of boundary-concepts, but not within the liberality of boundarylessness. (Sainsbury: 1990, 260) ${ }^{18}$

While vague concepts need not be bound, they can be. Furthermore, a competent judge is aware of this, as grasping a vague concept involves the realisation that "vagueness offers freedom [...] one may behave consistently with the nature of the concept in drawing a line between adjacent pairs". (Sainsbury: 1990, 259-60) It is this and not tolerance which is constitutive of vague predicates. ${ }^{19}$

Sainsbury raises two points of interest. First, he denies that tolerance governs competence with vague predicates: it does not follow from the boundarylessness of vague concepts that, for every $x$, if $x$ has been (competently) judged to satisfy a vague 
concept then, in order to retain competence, it is mandatory that its successor, $x^{\prime}$, if judged at all be judged likewise. What follows from the boundarylessness of a vague concept is that for every $x$, if $x$ has been judged to satisfy a vague concept then it is permissible that its successor, $x^{\prime}$, if judged at all be judged likewise. This brings us to the second point, Sainsbury's dictum that vagueness offers freedom.

Just how much freedom does vagueness offer? Clearly vagueness offers more freedom than many theorists have realised, the regularly adopted tolerance principle being overly restrictive. On the other hand, the competent use of vague predicates is not entirely unrestricted: there are clear-case and other penumbral constraints (Fine: $1975) .^{20}$

Suppose that the freedom emerges from two features. First, as Shapiro's open-texture principle above states, in borderline cases a speaker is free to assert ' $F x$ ' or ' $\neg F x$ ' without relinquishing competence. ${ }^{21}$ Second, as Sainsbury recognises, a judger is permitted to draw (non-legislative) boundaries between adjacent pairs and retain competence.

Accordingly, we can replace the principle of tolerance with the following dual principles which we can collectively refer to as principles of Permissible (In)tolerance:

Permissible Tolerance: For any non-clear (i.e. borderline) case $x$ in an ordered series, if $x$ is competently judged to satisfy $F$ then $x^{\prime}$ may be judged likewise, if judged at all. 
Permissible Intolerance: For any non-clear (i.e. borderline) case $x$ in an ordered series, if $x$ is competently judged to satisfy $F$ then $x^{\prime}$ may be judged to not satisfy $F$, if judged at all. ${ }^{22}$

Rather than stipulating ad hoc context shifts, we assume that the standard Kaplanian context <agent, location, world, time $>$ also contains a judge parameter. Variation in any parameter of the context marks a context shift. Most relevant for our purposes will be variation in the objects of the series (including their order of presentation) or in the judger of the context (either with a different judger or with the same judger on a different occasion).

The replacement of the tolerance principle with a permissibility principle allows us to give a minimal contextualist theory of vagueness without resorting to ad hoc context shifts. Call such a minimal theory Contextual Intolerance. ${ }^{23}$ The principles that form the basis of Contextual Intolerance are the assertion governing open-texture:

Open-texture: In borderline cases a subject is free to assert ' $\mathrm{Pa}$ ' and free to assert 'not-Pa', without offending against the meanings of the terms, or any other rules of language use.

A contextual version of the extension determining principle of judgementdependence:

Judgement-dependence (in context): An item in the borderline area lies in a given category, relative to context $c$ iff the relevant competent subject would judge it to lie in that category in context $c$. 
And our principles of Permissible (In)tolerance;

Permissible Tolerance: For any non-clear (i.e. borderline) case $x$ in an ordered series, if $x$ is competently judged to satisfy $F$ then $x$ ' may be judged likewise, if judged at all. ${ }^{24}$

Permissible Intolerance: For any non-clear (i.e. borderline) case $x$ in an ordered series, if $x$ is competently judged to satisfy $F$ then $x$ ' may be judged to not satisfy $F$, if judged at all.

Given Permissible (In)tolerance, and assuming the judgement dependence of vague predicates, the Universal Generalisation is false. It is simply not the case that if any object in a sorites series satifies a vague predicate an object which is relevantly similar will also be judged to satisfy the predicate; rather we say that if any object in a sorites series satifies a vague predicate an object which is relevantly similar may also be judged to satisfy the predicate. The context dependent nature of the judgementdependent principle determines that truth of such judgements is context dependent; the relevant propositions are true relative to a context of judgement. That is '\#938 is bald' may be true relative to judgement context $c$ but false relative to judgement context $c^{\prime}$. On this view context shifts are employed to accommodate the variable extensions of vague predicates, not to explain them away. ${ }^{25}$

Have we 'solved' the sorites? It is certainly the case that tolerance should be replaced by Permissible (In)tolerance. It is also the case that Permissible (In)tolerance does not entail the Universal Generalisation, unlike standard tolerance. But it is another 
question whether or not this satisfies us with regard to the paradox. The paradox has great appeal that is difficult to explain away. Below are considerations that take us at least some of the way towards this goal. ${ }^{26}$

The appeal of the paradox is partly explained by our propensity to confuse the lack of acontextual boundaries with a lack of contextual boundaries. Contextual Intolerance points out that that vague predicates permit context-dependent cut-offs. ${ }^{27}$ This differs from the claim that there is a cut-off such that that very cut-off holds in every context, which the Contextual Intolerance theorist denies. That is, we can accept the following contextualised version of the sharp boundaries claim, $(\exists c)(\exists x)\left(F x_{c} \& \neg F x^{\prime}{ }_{c}\right)$, and reject the acontextual version, $(\exists x)(\forall c)\left(F x_{c} \& \neg F x^{\prime}{ }_{c}\right)$.

It is also very plausible that the false mandatory tolerance principle;

Mandatory Tolerance: $\forall x J: F x \rightarrow$ (if judged at all) $M J: F x{ }^{\prime}$

(for every $x$, if $x$ has been competently judged to satisfy a vague predicate then, in order to retain competence, it is mandatory that its successor, $x^{\prime}$, if judged at all be judged likewise)

has been confused with the nearby true permissible tolerance principle;

Permissible Tolerance: $\forall x J: F x \rightarrow P J: F x$,

(for every $x$, if $x$ has been judged to satisfy a vague predicate then $x^{\prime}$ may be judged likewise).

These principles are in close cognitive proximity but only one of them leads us to paradox. 


\section{Conclusion}

Contextualist theories of vagueness have attempted to provide us with a package in which context dependent judgements and context shifts allow for preservation of the principle of tolerance and of the clear-case constraint. Unfortunately the context shifts that must be stipulated in order to preserve the principle of tolerance are entirely ad hoc and otherwise unmotivated. However, we can have something very close to the desirable package once we realise that it is a mistake to think that mastery of vague predicates is governed by a principle of tolerance. Rather mastery of vague predicates is governed by the (nearby) permissible (in)tolerance principle; a principle which does not lend itself to paradox. ${ }^{28}$ 


\section{REFERENCES}

\section{AUTHOR forthcoming XXX}

Fine, K. (1975) 'Vagueness, Truth and Logic', Synthese, 30: 265-300

Fara, D. Graff (2000) 'Shifting Sands: An Interest-Relative Theory of Vagueness', Philosophical Topics, 28: 45-81. Originally published under the name "Delia Graff".

Greenough, P. (2005) 'Contextualism about Vagueness and Higher-Order Vagueness', The Aristotelian Society, 79: 167-190

Greenough, P. and Ackerman, J. (2010) 'Hold the Context Fixed, Vagueness Still Remains' in R. Dietz and S. Moruzzi (eds.) Cuts and Clouds: Vaguenesss, its Nature and its Logic, (Oxford University Press: Oxford)

Gross, S. (2009) Review of Shapiro's 'Vagueness in Context', Philosophical Review, 118 (2): $261-266$

Lewis (1979) 'Scorekeeping in a Language Game', Journal of Philosophical Logic, 8: $339-59$

Raffman, D. (1994) 'Vagueness without Paradox', Philosophical Review, 103: 41-74

Sainsbury, R. M. (1991) 'Concepts without Boundaries', reprinted in R. Keefe \& P. Shapiro, S. (2003) 'Vagueness and Conversation' in J.C. Beall (ed.), Liars and Heaps (Oxford: Oxford University Press)

— (2006) Vagueness in Context (Oxford: Oxford University Press)

Stalnaker, R. (1999) 'Assertion' in R. Stalnaker, Context and Content, (Oxford: Oxford University Press)

Stanley, J. (2003) 'Context, interest-relativity, and the sorites', Analysis, 63: 269-80.

Wright, C. (1976) 'Language Mastery and the Sorites Paradox', in G. Evans and J. McDowell (eds.), Truth and Meaning: Essays in Semantics (Oxford: Oxford University Press)

\footnotetext{
${ }^{1}$ Where $x$ ' is a complex expression involving the bound variable $x$, equivalent to a description such as 'the successor of $x$ '.

${ }^{2}$ Note that there is a sense in which Graff Fara is the odd one out of the trio: for Shapiro and Raffman, weak tolerance allows us to claim that in no context is there a cut-off, for Graff Fara weak tolerance allows us to claim that the context-dependent cut-off can never be known.

${ }^{3}$ The particular challenges I present here are most relevant to Shapiro and Raffman's theories. For a detailed account of the ad hoc features of Graff Fara's contextualist theory see AUTHOR, forthcoming.
} 
${ }^{4}$ Notice that the claim that judgers will jump from 'bald' to 'not bald' judgements is a simplification; a reluctance to judge or a 'not sure' assertion is also classed as a jump.

${ }^{5}$ Shapiro states that such a borderline case is one in which the non-linguistic facts have not determined the truth-conditions for ' $F a$ '. However, as Greenough $(2005,170)$ notes, this is not what Shapiro should say as, for Shapiro, a vague predicate is one where the conditions of application are incomplete. That is, it is the linguistic facts and not the non-linguistic facts which fail to determine the truth conditions for ' $\mathrm{Fa}$ ' in a borderline case.

${ }^{6}$ See Gross (2009) for a discussion of the tension between tolerance and open-texture. Gross also clarifies that although Shapiro does not claim that tolerance is a part of a vague terms meaning it is consistent with his view that to maintain tolerance is a semantic default. $(2009,262$ : fn 1)

7 Shapiro borrows Raffman's psychological account of the phenomenon of backward spread as the basis of his semantic version. See Raffman (1994, 178-80).

${ }^{8}$ As an aside, the same criticism could be made of Lewis-why suppose that higher standards dominate lower standards? Why can lower standards not just remain fixed?

${ }^{9}$ It is true that the judger could not progress right through the series without switching predicates, otherwise he would judge at least one clear case incompetently, but that in itself does not justify backwards spread. It is the desire to preserve tolerance that motivates backwards spread.

${ }^{10}$ A desire to honour both the clear case constraints and the principle of tolerance is not a principled reason.

${ }^{11}$ See Stalnaker $(1999,54-5)$

${ }^{12}$ Notice, Shapiro is not claiming that explicit retraction is required for context change. The earlier assertion is removed when a jump occurs even if no retraction is made. Shapiro is simply motivating his theory by saying that the assertions should be removed because, if asked, the subject would retract his earlier assertion.

${ }^{13}$ This does not entail that the judger has a disposition to judge all of the objects to be the same colour; there is a gap between the disposition $\forall \mathrm{x}\left(\mathrm{J}: \mathrm{Fx} \rightarrow \mathrm{J}: \mathrm{Fx}^{\prime}\right)$ and the disposition $\mathrm{J}: \mathrm{Fa} \rightarrow \forall \mathrm{x}(\mathrm{J}: \mathrm{Fx})$.

14 Perhaps Shapiro can borrow Raffman's psychological, categoriser/discriminator distinction (see section 3 below and Raffman 1994, 47) but then tolerance stops being a semantic principle, as Shapiro requires it to be, and instead becomes a psychological principle.

${ }^{15}$ Shapiro might respond by claiming that he is committed to a view under which the scoreboard registers speaker's dispositions only and not the dispositional view of judgement that I propose on his behalf. However, as he is committed to vague predicates being judgement-dependent he would still be required to give some account of the relationship between dispositions and judgements. Note also that Shapiro commits himself to a form of judgement-dependence under which consensus of judgement is required and this certainly seems to be in tension with the dispositional account.

16 This same criticism can be put to Raffman.

${ }^{17}$ Notice that here the judger can justify his judgements by offering up the clear-case constraint.

${ }^{18}$ It is interesting that Delia Graff Fara (2000) uses tolerance and boundarylessness interchangeably in her theory of vagueness yet tolerance does not follow from boundarylessness.

${ }^{19}$ Note that, according to Sainsbury the fact that we are permitted to use vague predicates and draw sharp boundaries is not a feature of pragmatics: permissibility is a feature of the concept, not a pragmatic feature.

${ }^{20}$ A penumbral connection is a logical or conceptual relation holding among sentences that contain predicates with borderline cases. For example, something which is ' $F$-er' than something which satisfies 'is $F$ ' must itself satisfy 'is $F$ '; also, the same object cannot both satisfy and not satisfy 'is $F$ ' (within the same context). For more on penumbral connections see Fine (1975).

${ }^{21}$ It is perhaps worth raising a general worry here regarding the distinction between mandatory cases and judgement-dependent or permissible cases. It is claimed that vague predicates lack boundaries in that there is an area of permissibility. It does not follow from this claim that there is a switch of the direction of dependence. Shapiro claims that in the clear cases we act as 'detectivists', the clear cases determining our judgements, yet in the non-clear cases we act as 'projectivists', our judgements determining whether or not the predicate applies. It seems feasible that we could go 'projectivist' all the way, defining the clear cases as those which all competent judgers would judge to be clear cases as follows: $x$ is a clear case of $F$ iff for any subject meeting conditions $\mathrm{C}$, if the subject were to judge $x$, he would judge $F x$. Thinking of clear cases in this way seems to indicate that there could be a deeper connection between judgement-dependence and vague predicates but this is too big a digression to be taken up in this paper.

${ }^{22}$ Here and throughout we shall stipulate that judgements must reflect the ordering of the series thereby preserving monotonicity. 
23 The proposed theory is neutral on the question of whether standard contextualism, non-indexical contextualism, truth-relativism or content-relativism gives the correct semantics for language involving vague predicates. However, it is worth noting that any indexical theory, a theory under which content and not just truth value varies with context, will fall foul of Stanley's (2003) verb ellipses sorites.

${ }^{24}$ Note that the principle of permissible tolerance must range over pairs of borderline objects in order to make it compatible with the clear case constraint.

${ }^{25} \mathrm{We}$ are not always limited to reasoning pair by pair. Cannot a judge who judges equally two similar objects that are borderline cases of "F" reason correctly by universal generalisation and fall into the soritical conclusion? No. For the judge's dispositions have determined a cut off within the context making reasoning via universal generalisation faulty reasoning.

${ }^{26}$ Note that the diagnosis of the appeal of the sorites offered here (although not the solution) is perfectly analogous to that of the supervaluationist. (See Fine, 1975).

${ }^{27}$ In this respect Contextual Intolerance is similar to Graff Fara's (2000) view.

${ }^{28}$ Thanks to an anonymous referee for this journal whose comments led to vast improvements of the paper in places and aspects too numerous to mention individually. 